\title{
An opaque Sun? \\ The potential for future, higher opacities to solve the solar abundance problem
}

\author{
Regner Trampedach ${ }^{1,2, \star}$ \\ ${ }^{1}$ Space Science Institute, 4750 Walnut Street, Suite 205, Boulder, CO 80301 USA \\ ${ }^{2}$ Stellar Astrophysics Centre, Dept. of Physics and Astronomy, Ny Munkegade 120, Aarhus University, DK-8000 Aarhus C, \\ Denmark
}

\begin{abstract}
Last year Bailey et al. announced their measurement of iron opacity that increases the Rosseland mean at the base of the solar convection zone by $7 \%$. I ask what happens if the absorption by other elements is also stronger than predicted so far.

Artificially increasing the absorption by other elements, proportional to the number of bound electrons in the absorber (reflecting our remaining ignorance of atomic physics) gives an opacity increase for a solar model, that has the potential to solve the long-standing solar abundance problem.

Conclusion: Opacities are the likely source of the solar abundance problem, and the solar abundances are likely closer to those of Asplund et al. (2009) than to the various classic sets of abundances.
\end{abstract}

\section{Introduction}

Solar abundances, as derived from spectra originating in the photosphere, are used as the reference for stellar abundances. Stars are usually assumed to have individual $\mathrm{H}$ and He abundances, but to have the same mixture of metals as does the Sun. This means that solar abundance determinations can affect our interpretation of a major part of astronomical observations.

A number of solar abundance analyses were published in the 1990's and form the classic solar abundances, e.g., [1-4] and [5] (the latter, from hereon GN93). They were based on 1D semi-empirical atmosphere models, e.g., [6].

The modern solar abundances, based on 3D simulations of deep, convective, radiation-coupled, solar atmospheres were first published by [7], challenged by [8] and [9] (from hereon CLSFB11) and updated by [10] (from hereon AGSS09). The latter were confirmed by improved analysis of the same solar 3D convection simulation, but with improved atomic physics and statistical analysis by [11-13] (from hereon SGA15). The crucial light elements are still awaiting a similar re-analysis. The differences from GN93 to AGSS09 and SGA15 are shown in Fig. 1.

The AGSS09 abundances constitute a reduction of C, $\mathrm{N}$ and $\mathrm{O}$ to about $70 \%$ of the classic GN93 values. In particular oxygen contributes significant opacity from the bottom of the solar convection zone and inward, so the reduction in $[\mathrm{O} / \mathrm{H}]$ changes the interior structure of our solar models and moves the bottom of the convective envelope closer to the surface by many standard deviations.

This disrupts the previous agreement between models and helioseismic inversions, as is well documented

\footnotetext{
^e-mail: rtrampedach@SpaceScience.org
}

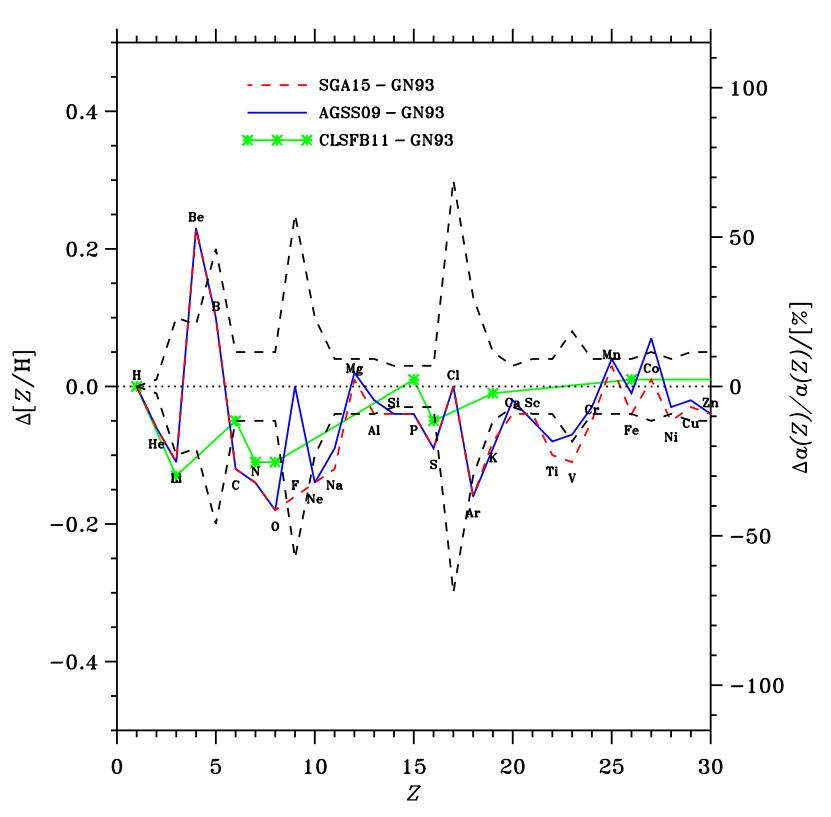

Figure 1: Some modern solar abundance determinations, relative to the 'classic' GN93 abundances. The dashed black lines show the error-bars of the SGA15 abundances, making everything outside, significantly different from the classic GN93 abundances, with $\mathrm{C}, \mathrm{N}$ and $\mathrm{O}$ constituting the most significant difference. AGSS09 (blue) and SGA15 (red dashed) are very similar, whereas CLSFB11 (green) is closer to the classic mixture.

by, e.g., [14, 15]. This is the so-called solar abundance problem. Many solutions to this problem have been proposed [16-20], but none of them have succeeded in achiev- 
ing simultaneous agreement with the seismically inferred helium abundance and depth of the convection zone, as demonstrated by [21].

It is natural to question the $3 \mathrm{D}$ solar atmosphere simulations that precipitated this disagreement with helioseismology. We did [22], and found that the simulations exhibit an unprecedented simultaneous agreement with a wide range of solar observations, including continuum limb-darkening, spectral flux distribution, the $\mathrm{H} \alpha, \beta$ and $\mathrm{Pa} \beta, \gamma$ lines of hydrogen. Even the spatially (granulescale) resolved oxygen lines (including non-LTE effects) match observations $[23,24]$. This rather remarkable, considering the lack of parameters that are free to fit any of these results.

It should also be pointed out that [25] argue strongly against using the classic solar abundances, with Grevesse \& Sauval being authors of both this rebuttal, many of the classic abundance determinations, as well as AGSS09. [25] found the main differences between the classic and AGSS09 abundances to be largely due to improved atomic line-parameters, the homogeneous analysis carried out by AGSS09, increased computer-power for non-LTE calculations and the much improved detection of blends due to the simulation's good agreement with the detailed Cshaped line-profiles. Direct 3D effects of the simulations are rather small except for molecular lines.

\section{Opacities and Abundances}

\subsection{Opacity Solutions to the solar abundance problem}

Only two viable solutions to the solar abundance problem, has bee found so far: Change the abundances back to the classic values, or increase the opacity below the solar convection zone. [26] determined the profile of opacity enhancements that would make a solar model with AGSS09 abundances have the same structure as Model S by [27]. This standard solar model was evolved with the classic GN93 abundances (dashed, black curve in Fig. 2, marked ' $f_{\kappa}(\mathrm{AGSS} 09)$ '). The implication being that such a modified AGSS09 model would show similar helioseismic agreement as the highly successful Model S. A similar result was found by [28] (dot-dashed, black curve in Fig. 2, marked ' $f_{\kappa}(\mathrm{AGS} 05) / 2$ '). Whether such opacity increases were but wishful thinking, was not known at the time.

\subsection{Laboratory Experiments}

[29] (from hereon B15) carried out the impressive feat of measuring an iron plasma at conditions close to those at the bottom of the solar convection zone: a temperature of $T=2.11 \times 10^{6} \mathrm{~K}$ and electron density of $N_{\mathrm{e}}=3.1 \times$ $10^{22} \mathrm{~cm}^{-3}$. This $N_{\mathrm{e}}$ is still about three times lower than the solar value at that temperature, though. B15 measured the transmission spectra of the plasma (predominantly $\mathrm{Fe}^{16+}-$ $\mathrm{Fe}^{18+}$ ) and computed the underlying absorption coefficient (ensuring that deviations from local thermodynamic equilibrium, homogeneity and stationarity were insignificant). This absorption coefficient is generally larger, and has broader features than any current opacity calculations. Specifically, compared to the [30] (OP05) Fe opacity at the same conditions, the observed ones are 1.5-1.8 times larger in the rather featureless, short-wavelength, continuum below $9.5 \AA$. In the minima between lines (the part that determines the harmonic Rosseland mean) the observed absorption is 3-5 times stronger. B15 did the experiment of calculating the OP05 Rosseland mean for a full solar mixture, and found that substituting the theoretical by the observed Fe absorption, increased this opacity by $7 \pm 3 \%$. This is a substantial amount, and far from trivial to explain theoretically [31]. And it is about half of the opacity increase needed to reconcile an AGSS09 solar model with helioseismology.

\subsection{OP05 Opacity Experiments}

If the experimental results on iron are not somehow a special property of iron, then the absorption by other elements should also be larger than predicted by current calculations. Experiments are therefore carried out on the OP05 absorption coefficients of individual elements, summed up over the AGSS09 mixture and Rosseland averaged along the fixed $\varrho, T$-stratification of Model S [27].

The first step is to broaden the Fe absorption to make it qualitatively similar to Fig. 3 of B15, as shown with blue dotted line in Fig. 2. The next step is a wavelength independent enhancement factor on Fe, calibrated to give the observed $7 \%$ increase in Rosseland opacity of the full mixture (blue solid in Fig. 2). This is a crude but simple simulation of B15's results. More than qualitative agreement cannot be expected, as the present work does not offer an improved opacity calculation, but merely asks whether such improved opacity calculations are likely to solve the solar abundance problem, given the B15 experiment.

The next and crucial step is to extrapolate from iron to the 16 other elements included in OP05. We know the simple hydrogen- and helium-like ions very well, and it would be hard to argue for any phenomena that could increase their absorption. One- and two-electron systems are therefore kept at their current theoretical level of absorption. This already gives an electron dependency of the absorption enhancements, up to the 8-10 electron Fe ions, present in the plasma of the B 15 experiment. A linear dependency is favoured for its simplicity. Whether to use the number of bound electrons in the absorber, or the number of closed electron shells (as motivated in Sect. 2.4 and shown with solid magenta in Fig. 2), has little effect on the final opacity increase. The opacity enhancements shown with solid lines in Fig. 2 also include a slight increase with $\log T$, designed to give no enhancement at $\log T=5$. This is seen to give good agreement with the needed enhancement found by [26] (dashed black curve). Having no $T$-dependency makes it closer to the needed enhancement found by [28]. Either way, the artificial opacity enhancement, calibrated against the B15 experiment, has the correct shape and magnitude to solve the solar abundance problem. 


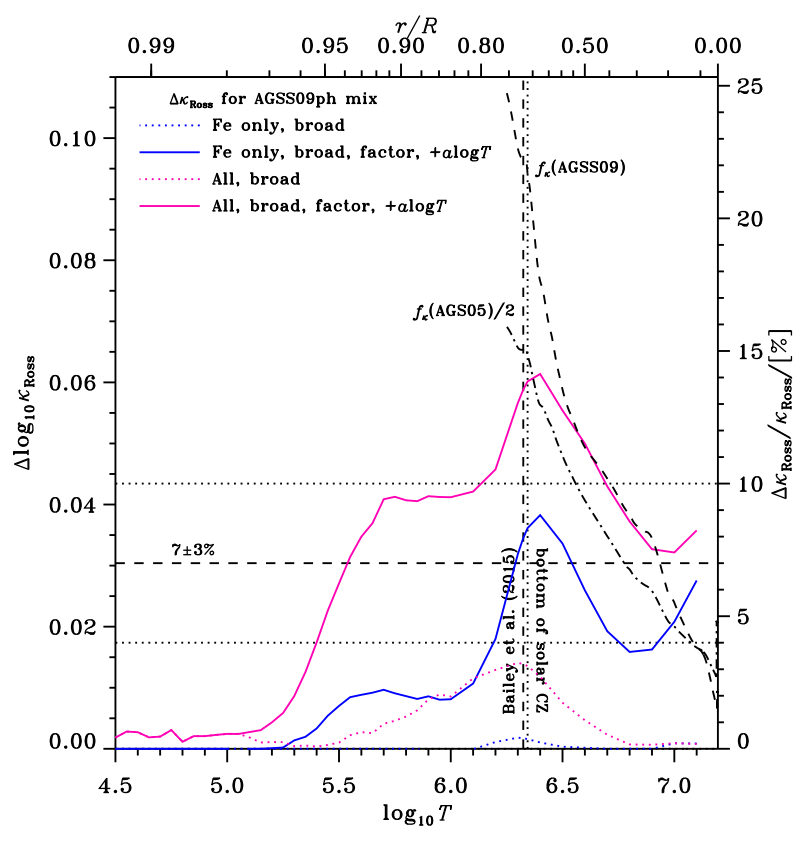

Figure 2: Opacity enhancements, $\Delta \kappa_{\text {Ross }}$, along the fixed $\varrho, T$ stratification of standard solar Model S [27], as $\Delta \log \kappa$ on the left-hand scale and fractional change on the right-hand scale. Position in the model is shown both on the temperature scale, $\log T$ (bottom), and the fractional radius, $r / R$-scale (top). Blue curves shows only Fe enhanced, calibrated to the experimental result (horizontal and vertical dashed lines), and magenta curves show result of artificially enhancing the absorption by all elements. Dotted lines show the effect of broadening only, and solid lines also include the enhancement according to the number of closed electronic shells in the absorbing ions. The dashed, black curve marked ' $f_{\kappa}$ (AGSS09)' is the opacity enhancement found by [26] to restore agreement with helioseismology, and the dotdashed curve marked ' $f_{\kappa}($ AGS05)/2' is that found by [28].

\subsection{New Opacity Calculations}

Recently OPAS-team, [32], and the Next Gen Los Alamos opacity-team, [33], published their newest opacity calculations, aimed at solving the solar abundance problem. They only obtained $13-25 \%$ of the increase needed to restore agreement, however, again demonstrating how persistent the problem is.

New calculations by [34] has looked at how core electrons can increase the absorption. It has usually been assumed that only the outermost, valence electrons, interacts with the radiation field. Radiation is so intense at these temperatures, however, that the electrons of filled shells can be excited, often together with a valence electron. This gives rise to a whole new absorption system of continua and lines. Some of these processes were included in approximate ways for the OP05 calculations. The new calculation by [34] on the other hand, dispenses with some crucial approximations, and also check for completeness in terms of configurations included in the parent and target states. Their impressive calculation seems converged when including up to $n=4$ complexes of the target ion, and gives continuum enhancements of orders of magni- tude for absorption from individual states of the parent ion (although only a 9\% increase for ground-state absorption), and in the right wavelength region to explain B15's results.

\section{Conclusions}

Solar abundance analysis based on realistic 3D convective atmosphere simulations, result in lower $\mathrm{C}, \mathrm{N}$ and $\mathrm{O}$ abundances. This in turn makes for solar models that disagree with helioseismology by many standard deviations, known as the solar abundance problem (see Sect. 1). It has been shown that agreement can be restored if the opacities are artificially increased by $20 \%$ at the bottom of the convection zone, down to a few percent increase at the centre (see Sect. 2.1). Whether such increases in opacity were realistic was unknown at the time.

The experiments by B15 directly measured the iron absorption at conditions close to those at the bottom of the solar convection zone, and found larger and broader absorption than any theoretical calculations to date.

Performing experiments on the OP05 absorption coefficients, artificially enhanced and broadened to match the iron experiment, gives an increase of the Rosseland mean of the AGSS09 mixture, that has the potential to solve the solar abundance problem. The enhancement was extrapolated from iron, by scaling it by the number of filled electronic shells (see Sect. 2.3), reflecting our remaining ignorance of atomic physics, as well as the evidence for excited core electrons being the culprit.

And the theoretical work by [34], on absorption by core electrons in filled shells, does indeed point to this being the missing absorption in B15's experiments (see Sect. 2.4).

New physics in the opacity calculations therefore seems to be the likely solution to the solar abundance problem - a problem that turned out not to have much to do with abundances after all, but rather is an unfortunate conspiracy of nature and the historic events that lead to the classic abundances - confirming the old adage that you can have the correct results for the wrong reasons.

The artificial opacity enhancements, presented here, should not be used for computing new opacity tables to be applied to stellar evolution calculations. This is merely an exercise to answer the question, whether improved opacity calculations can realistically solve the solar abundance problem, and the affirmation will hopefully encourage the efforts of the various teams working on such opacity calculations. It will have a broad impact on a large section of solar and stellar modelling, asteroseismology, Galactic evolution, etc.

These changes to stellar opacities will fortunately not affect the atmospheres of solar-like stars, and there will therefore not be a feed-back into the solar abundance determination. The two are safely decoupled. The author recommends the AGSS09 abundances, supplanted by the [11-13] for all but the light elements, as the most likely, present day composition of the solar atmosphere and convective envelope. 


\section{Acknowledgements}

RT acknowledges funding from NASA grant NNX15AB24G. Funding for the Stellar Astrophysics Centre is provided by The Danish National Research Foundation (grant DNRF106). This research has made extensive use of NASA's Astrophysics Data System.

\section{References}

[1] E. Anders, N. Grevesse, Geochim. Cosmochim. Acta 53, 197 (1989)

[2] N. Grevesse, Solar Abundances: the Reference System, in Evolution of Stars: the Photospheric Abundance Connection, edited by G. Michaud, A.V. Tutukov (Kluwer, Dordrecht, 1991), Number 145 in IAU Symp., pp. 63-69

[3] N. Grevesse, A. Noels, A.J. Sauval, Standard abundances, in Cosmic Abundances, edited by S.S. Holt, G. Sonneborn, ASP (ASP, San Francisco, 1996), Vol. 99 of Conf. Ser., pp. 117-126

[4] N. Grevesse, A.J. Sauval, Space Sci. Rev. 85, 161 (1998)

[5] N. Grevesse, A. Noels, Cosmic abundances of the elements, in Origin and Evolution of the Elements, edited by N. Prantzos, E. Vangioni-Flam, M. Cassé (Cambridge Univ. press, 1993), pp. 15-25

[6] H. Holweger, E.A. Müller, Sol. Phys. 19, 19 (1974)

[7] M. Asplund, N. Grevesse, J. Sauval, The solar chemical composition, in Cosmic abundances as records of stellar evolution and nucleosynthesis, edited by T.G. Barnes III, F.N. Bash (Astron. Soc. Pac., San Francisco, 2005), Number 336 in ASP Conf. Ser., pp. 25-38

[8] K. Lodders, ApJ 591, 1220 (2003)

[9] E. Caffau, H.G. Ludwig, M. Steffen, B. Freytag, P. Bonifacio, Solar Physics 268, 255 (2011)

[10] M. Asplund, N. Grevesse, A.J. Sauval, P. Scott, ARA\&A 47, 481 (2009)

[11] P. Scott, N. Grevesse, M. Asplund, A.J. Sauval, K. Lind, Y. Takeda, R. Collet, R. Trampedach, W. Hayek, A\&A 573, A25:1 (2015)

[12] P. Scott, M. Asplund, N. Grevesse, M. Bergemann, A.J. Sauval, A\&A 573, A26:1 (2015)

[13] N. Grevesse, P. Scott, M. Asplund, A.J. Sauval, A\&A 573, A27:1 (2015)

[14] J.N. Bahcall, S. Basu, M.H. Pinsonneault, A.M. Serenelli, ApJ 618, 1049 (2005)
[15] H.M. Antia, S. Basu, ApJ 620, L129 (2005)

[16] J.J. Drake, P. Testa, Nature 436, 525 (2005)

[17] J.A. Guzik, L.S. Watson, A.N. Cox, ApJ 627, 1049 (2005)

[18] A.C. Vincent, P. Scott, R. Trampedach, MNRAS 432, 3332 (2013)

[19] J.A. Guzik, K. Mussack, ApJ 713, 1108 (2010)

[20] A.M. Serenelli, W.C. Haxton, C. Peña-Garay, ApJ 743, 24:1 (2011)

[21] F. Delahaye, M.H. Pinsonneault, ApJ 649, 529 (2006)

[22] T.M.D. Pereira, M. Asplund, R. Collet, I. Thaler, R. Trampedach, J. Leenaarts, A\&A 554, A118:1 (2013), 1304.4932

[23] T.M.D. Pereira, D. Kiselman, M. Asplund, A\&A 507, 417 (2009)

[24] T.M.D. Pereira, M. Asplund, D. Kiselman, A\&A 508, 1403 (2009)

[25] N. Grevesse, M. Asplund, A.J. Sauval, P. Scott, Why GN93 should not be used anymore, in Ageing Low Mass Stars: From Red Giants to White Dwarfs, edited by J. Montalbán, A. Noels, V. van Grootel (2013), Vol. 43 of European Physical Journal Web of Conferences, pp. 01004:1-3

[26] J. Christensen-Dalsgaard, G. Houdek, Ap\&SS 328, 51 (2010)

[27] J. Christensen-Dalsgaard, W. Däppen, S.V. Ajukov, E.R. Anderson, H.M. Antia, S. Basu, V.A. Baturin, G. Berthomieu, B. Chaboyer, S.M. Chitre et al., Science 272, 1286 (1996)

[28] A.M. Serenelli, S. Basu, J.W. Ferguson, M. Asplund, ApJ 705, L123 (2009)

[29] J.E. Bailey, T. Nagayama, G.P. Loisel, G.A. Rochau, C. Blancard, J. Colgan, P. Cossé, G. Faussurier, C.J. Fontes, F. Gilleron et al., Nature 517, 56 (2015)

[30] N.R. Badnell, M.A. Bautista, K. Butler, F. Delahaye, C. Mendoza, P. Palmeri, C.J. Zeippen, M.J. Seaton, MNRAS 360, 458 (2005)

[31] C.A. Iglesias, High Energy Density Physics 15, 4 (2015)

[32] M. Le Pennec, S. Turck-Chièze, S. Salmon, C. Blancard, P. Cossé, G. Faussurier, G. Mondet (2015), 1510.05600

[33] J. Colgan, D.P. Kilcrease, N.H. Magee Jr., M.E. Sherrill, J. Abdallah, Jr., P. Hakel, C.J. Fontes, J.A. Guzik, K. Mussack (2016), 1601.01005

[34] S.N. Nahar, A.K. Pradhan, Phys. Rev. Lett. 116, 235003:1 (2016) 